\title{
A novel small molecule compound VCP979 improves ventricular remodeling in murine models of myocardial ischemia/reperfusion injury
}

\author{
JING LIU ${ }^{1-3}$, QINGSHU MENG ${ }^{2,4}$, XIAOTING LIANG ${ }^{2,4}$, RULIN ZHUANG ${ }^{2,3}$, \\ DONGSHENG YUAN ${ }^{2,3}$, XINYU GE ${ }^{2,3}, \mathrm{HAO} \mathrm{CAO}^{3}$, FANG LIN $^{2,4}, \mathrm{XIN} \mathrm{GONG}^{5}$, \\ HUIMIN FAN $^{2-5}$, BINGHUI WANG ${ }^{6,7}$, XIAOHUI ZHOU ${ }^{2,4}$ and ZHONGMIN LIU $^{1-5}$ \\ ${ }^{1}$ Shanxi Medical University, Taiyuan, Shanxi 030001; ${ }^{2}$ Research Center for Translational Medicine; \\ ${ }^{3}$ Department of Cardiovascular and Thoracic Surgery, Shanghai East Hospital, Tongji University School of Medicine; \\ ${ }^{4}$ Shanghai Heart Failure Research Center, ${ }^{5}$ Department of Heart Failure, Shanghai East Hospital, \\ Tongji University School of Medicine, Shanghai 200120, P.R. China; ${ }^{6}$ Biomarker Discovery Laboratory, \\ Baker Heart and Diabetes Institute; ${ }^{7}$ Monash Centre of Cardiovascular Research and Education in Therapeutics, \\ Department of Epidemiology and Preventive Medicine, Monash University, Melbourne, Victoria 3004, Australia
}

Received June 26, 2019; Accepted October 25, 2019

DOI: $10.3892 /$ ijmm.2019.4413

\begin{abstract}
Persistent ventricular remodeling following myocardial ischemia/reperfusion (MI/R) injury results in functional decompensation and eventual progression to heart failure. VCP979, a novel small-molecule compound developed in-house, possesses anti-inflammatory and anti-fibrotic activities. In the present study, no significant pathological effect was observed following the administration of VCP979 on multiple organs in mice and no difference of aspartate transaminase/alanine aminotransferase/lactate dehydrogenase levels was found in murine serum. Treatment with VCP979 ameliorated cardiac dysfunction, pathological myocardial fibrosis and hypertrophy in murine MI/R injury models. The administration of VCP979 also inhibited the infiltration of inflammatory cells and the pro-inflammatory cytokine expression in hearts post MI/R injury. Further results revealed that the addition of VCP979 prevented the primary neonatal cardiac fibroblasts (NCFs) from Angiotensin II (Ang II)-induced collagen synthesis and neonatal cardiac myocytes (NCMs)
\end{abstract}

Correspondence to: Professor Zhongmin Liu, Shanxi Medical University, 85 South Jie Fang Road, Taiyuan, Shanxi 030001, P.R. China

E-mail: liu.zhongmin@tongji.edu.cn

Professor Xiaohui Zhou, Shanghai Heart Failure Research Center, Shanghai East Hospital, Tongji University School of Medicine, 150 Jimo Road, 14-D2, Pudong, Shanghai 200120, P.R. China E-mail: zxh100@tongji.edu.cn

Key words: VCP979, MI/R injury, ventricular remodeling, p38-MAPK, inflammation hypertrophy. In addition, VCP979 attenuated the activation of p38-mitogen-activated protein kinase in both Ang II-induced NCFs and hearts subjected to MI/R injury. These findings indicated that the novel small-molecule compound VCP979 can improve ventricular remodeling in murine hearts against $\mathrm{MI} / \mathrm{R}$ injury, suggesting its potential therapeutic function in patients subjected to MI/R injury.

\section{Introduction}

Ischemic heart disease is one of the most common causes of morbidity and disability worldwide (1). Timely and effective myocardial reperfusion by percutaneous coronary intervention is essential to salvage reversible injuries in the ischemic myocardium (2). However, reperfusion may paradoxically aggravate the tissue damage by promoting myocardial death, cardiac dysfunction, ventricular remodeling and the inflammatory response, all of which fall under myocardial ischemia/reperfusion $(\mathrm{MI} / \mathrm{R})$ injury $(2,3)$. Short-term hypertrophic and fibrotic response is adaptive and protective, yet persistent pathological hypertrophy and excessive fibrosis accelerates the progression of ventricular remodeling following MI/R injury (4). Currently, the cardioprotective medicines available for the treatment of patients with heart failure, including vasodilators, loop diuretics and inotropic agents, can alleviate congestion and improve hemodynamics short-term. However, few drugs are capable of preventing end-organ damage or improving long-term outcomes (5). Therefore, it is of vital importance to explore novel therapeutic options to alleviate ventricular remodeling and prevent progression to heart failure.

Previous preclinical and clinical studies have shown that numerous small-molecule compounds can prevent myocardial cell death during ischemia and subsequent reperfusion $(6,7)$. It has been reported that small molecule drugs with a low molecular weight show good spatial dispersion in vivo and 
are easier to absorb than multiple protein-based drugs, thus exerting obvious effects on numerous pathological processes (8). Small-molecule compounds may therefore lead to the development of new therapeutic agents.

Previous evidence has revealed that MI/R injury is associated with the activation of the p38-mitogen-activated protein kinase (MAPK) signaling pathway in murine models. The suppression of p38-MAPK decreases the production of inflammatory cytokines, including interleukin (IL)-1, IL-8 and tumor necrosis factor- $\alpha$ (TNF- $\alpha)$, suggesting the therapeutic potential of p38-MAPK inhibitors in ischemic heart disease $(9,10)$ However, the classic p38 inhibitor SB203580 has a low specificity during other protein kinase functions (11). VCP979, a small-molecule compound with novel chemical pharmacophores, was developed by the authors' colleagues at the Monash University as a novel anti-fibrotic and anti-inflammatory agent (12). VCP979 was developed as a selective inhibitor of p38-MAPK. The authors' preliminary studies also indicated that VCP979 possesses a p38-MAPK inhibitory activity and has significant anti-fibrotic effects in vitro and in vivo $(11,13)$. In the diabetic murine model, VCP979 can decrease the infarct volume and promote ischemic stroke recovery and axonal/WM remodeling (11). However, whether its administration can protect the heart from MI/R injury remains unclear. The aim of the present study was to determine the role of the novel small-molecule compound VCP979 in cardiac protection post $\mathrm{MI} / \mathrm{R}$ injury and explore the possible underlying mechanism.

\section{Materials and methods}

Animals. The 6-8 weeks old male C57BL/6 mice (22-26 g; $\mathrm{n}=60)$ and neonatal Sprague-Dawley (SD) rats $(6-7 \mathrm{~g} ; \mathrm{n}=35)$ were purchased from Shanghai SLAC Laboratory Animal Co., Ltd. In the whole experimental process, mice were bred at $20-25^{\circ} \mathrm{C}$ at 12 -h light/dark cycles and given food and water freely under specific pathogen-free conditions. The experimental procedures were implemented following the approval of the Institutional Animal Care and Use Committee of Tongji University (Shanghai, China; approval no. TJLAC-019-121).

$M I / R$ injury model establishment. Briefly, mice were anesthetized with sodium pentobarbital $[60 \mathrm{mg} / \mathrm{kg}$, intraperitoneal (i.p.) injection]. During surgery, a heating pad was used to maintain the animal temperature at $37.5-38.5^{\circ} \mathrm{C}$. Next, mice were intubated and mechanically ventilated using a Harvard rodent respirator. A left thoracotomy at the 4-5th intercostal space was performed and the left anterior descending coronary artery (LAD) was transiently ligated under $2 \mathrm{~mm}$ of lower margin of the left auricle with an 8-0 suture, as previously described (14). The ligation was successful when the anterior wall of left ventricle turned white. The MI/R groups were subjected to $45 \mathrm{~min}$ ischemia, then reperfusion until day 28. The sham mice underwent the same surgical procedure, except the LAD suture was not tightened. Then, at 1 day after surgery, the cardiac function of each group was detected respectively and mice with left-ventricular ejection fraction (LVEF) below 50\% were considered successful MI/R models. Following surgery, mice were treated with normal saline (N.S) and VCP979 (50 mg/kg/day) via an i.p. injection for 14 consecutive days.
VCP979 administration protocol. The small-molecule compound VCP979 was stored as a powder and dissolved in dimethyl sulfoxide initially, and then further diluted in $0.9 \%$ N.S immediately before use.

All mice were randomly assigned to four groups (6 mice per group): i) Sham group injected with N.S; ii) sham group injected with VCP979; iii) MI/R group injected with N.S; and iv) MI/R group injected with VCP979. The treatment protocol is shown in Fig. 1. Each experiment was repeated at least three times.

Echocardiography. Transthoracic two-dimensional echocardiography using a Vevo 2100 high-resolution imaging system with a $30-\mathrm{MHz}$ linear transducer (FUJIFILM Visual-Sonics, Inc.) was performed to assess before and after MI/R injury with $2 \%$ isoflurane inhalation. The cardiac function of all animals was evaluated through long-axis scans using M-mode images including LVEF, left-ventricular fractional shortening (LVFS), left-ventricular volume; diastolic/systolic (LV Vol; $\mathrm{d} / \mathrm{s}$ ) and left-ventricular internal dimension; diastolic/systolic (LVID; d/s) (15). Cardiac function in each group was detected 1 day before and 1, 7, 14 and 28 days after surgery.

The safety detection of VCP979 in mice. The blood ( 1 ml) of sham groups was collected 14 days after N.S or VCP979 injection and the serum was isolated by centrifugation $(840 \mathrm{x} \mathrm{g}$, $4^{\circ} \mathrm{C}, 10 \mathrm{~min}$ ). Aspartate transaminase (AST), alanine aminotransferase (ALT) and lactate dehydrogenase (LDH) levels were measured using Beckmann AU680.

Cell culture and treatment. Neonatal cardiac myocytes (NCMs) and neonatal cardiac fibroblasts (NCFs) were isolated from the hearts of 1-3-day-old SD rat pups as previously described (16). The non-adherent NCMs were separated from the NCFs after $90 \mathrm{~min}$ of incubation. The NCMs and NCFs were cultured in high-glucose DMEM (Gibco; Thermo Fisher Scientific, Inc.) supplemented with $10 \%$ fetal bovine serum (Gibco; Thermo Fisher Scientific, Inc.), BrdU (100 nM, only added in the NCMs culture medium), $1 \%$ penicillin $(100 \mathrm{U} / \mathrm{ml})$ and $1 \%$ streptomycin $(100 \mu \mathrm{g} / \mathrm{ml})$, and were incubated at $37^{\circ} \mathrm{C}$ in a humidified atmosphere of $95 \%$ air and $5 \% \mathrm{CO}_{2}$. In addition, NCMs and NCFs were stimulated with Angiotensin II (Ang II; Sigma-Aldrich; Merck KGaA) $200 \mathrm{nM}$ for $24 \mathrm{~h}$, and then treated with different concentrations of VCP979 $(0.1,1,3$ and $9 \mu \mathrm{M}$ ) for another $24 \mathrm{~h}$.

Cell supernatants $\mathrm{LDH}$ release assay. The $\mathrm{LDH}$ release to the medium was detected using an LDH CytoTox 96 reagent kit (Promega Corporation), according to the manufacturer's protocol. In a 96-well plate, $50 \mu \mathrm{l}$ cell supernatants were mixed with $50 \mu$ l CytoTox 96 reagents and incubated at room temperature for $30 \mathrm{~min}$. Finally, $50 \mu \mathrm{l}$ stop solutions was added and the absorbance value was measured at $490 \mathrm{~nm}$ using a microplate spectrophotometer (M200pro; Tecan Group, Ltd.).

Measurement of cell proliferation. Cell proliferation was evaluated using a Cell Counting Kit-8 (CCK-8) assay (Beijing Solarbio Science \& Technology Co., Ltd.). The NCFs were plated on 96-well plates at a density of $1 \times 10^{4}$. Following stimulation with AngII (200 nM) for $24 \mathrm{~h}$, the NCFs were treated 


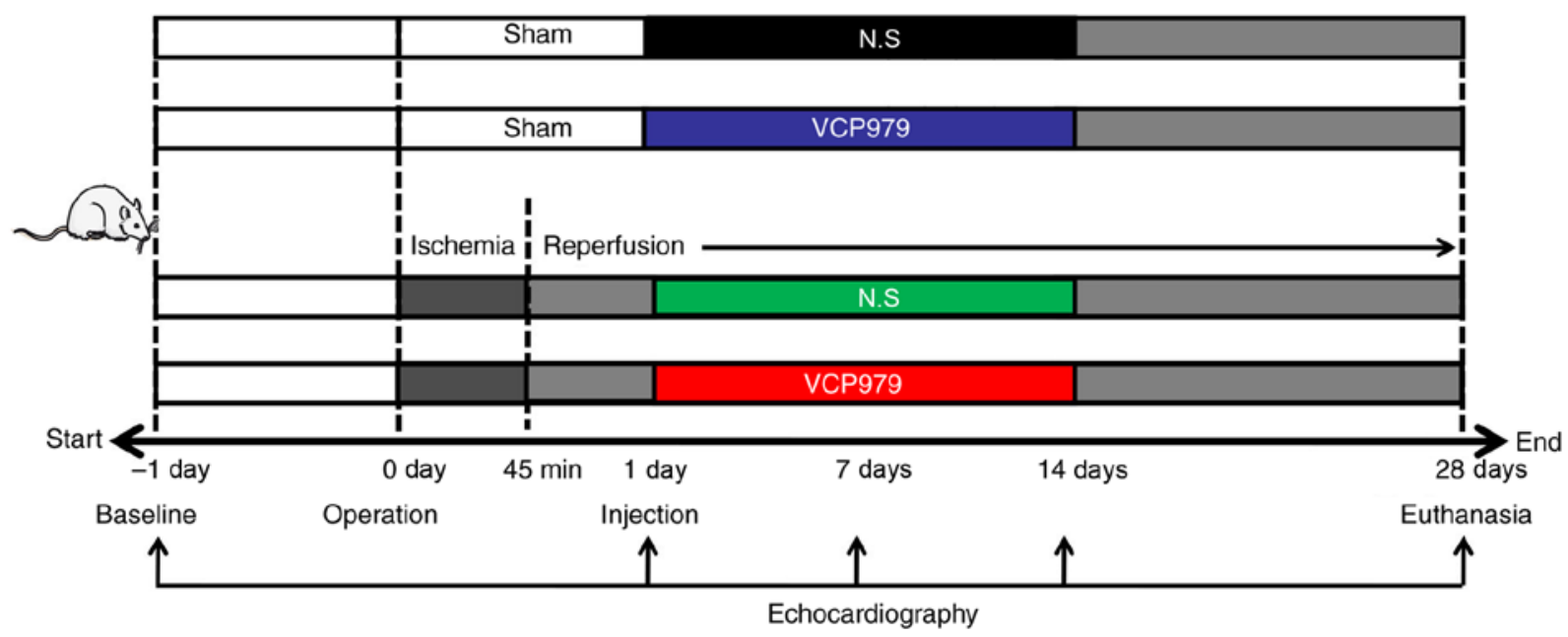

Figure 1. Schematic representation of the experimental protocol for murine models. The four groups: Sham+N.S, sham+VCP979, MI/R+N.S and MI/R+VCP979 ( $\mathrm{n}=6$ per group). MI/R injury was established by $45 \mathrm{~min}$ ischemia and continuous reperfusion until day 28 . VCP979 and N.S were injected intraperitoneally from days 1 to 14 following surgery. The experiments were repeated three times. MI/R, myocardial ischemia/reperfusion; N.S, normal saline.

with or without different concentrations of VCP979 $(0.1,1,3$ and $9 \mu \mathrm{M}$ ) for another $24 \mathrm{~h}$. According to the manufacturer's protocol, $10 \mu \mathrm{l}$ CCK-8 solution was added to each well and the cells were incubated at $37^{\circ} \mathrm{C}$ for $1-2 \mathrm{~h}$. A microplate spectrophotometer (M200pro) was used to read the optical density of each well at $450 \mathrm{~nm}$.

Immunofluorescence detection. Cardiomyocyte hypertrophy was determined using TRITC-Phalloidin (Beijing Solarbio Science \& Technology Co., Ltd.) staining, following the manufacturer's protocol. Briefly, the cells $\left(5 \times 10^{5}\right.$ cells/well $)$ were plated on 6-well plates and fixed with $4 \%$ paraformaldehyde (PFA) for $10 \mathrm{~min}$ at room temperature. Following $0.5 \%$ Triton X-100 permeability treatment, the NCMs were stained with TRITC-Phalloidin (1:50) for $30 \mathrm{~min}$ at room temperature in the dark, whereas the nucleus was stained with 4 ', 6-diamidino-2-phenylindole [room temperature (RT), $10 \mathrm{~min}$ ]. Phalloidin can label the F-actin of the cytoskeleton with high specificity and sensitivity. After staining with Phalloidin, the ImageJ software 1.49p (National Institutes of Health) was used to measure the cytoskeleton of each cell. A total of 5 different fields were randomly selected and analyzed under an upright fluorescent microscope (Leica DM2500; Leica Microsystems $\mathrm{GmbH})$.

Histological analysis. The animals were euthanized on day 14 or 28 after surgery. The tissues were dissected and fixed $\left(4^{\circ} \mathrm{C}\right)$ in $4 \%$ PFA overnight, embedded in paraffin, sectioned into $4-\mu \mathrm{m}$ slices and then stained (RT, $5 \mathrm{~min}$ ) with hematoxylin and eosin (H\&E) and Masson's Trichrome. The relative myocyte cross-sectional areas (CSAs) were detected by wheat germ agglutinin (WGA; Abcam) conjugated to Alexa Fluor 488 (1:200; Thermo Fisher Scientific, Inc.), as previously described (17) and imaged using a confocal microscope (Leica SP8; Leica Microsystems, Inc.). In addition, myocardial fibrosis was identified by immunohistochemistry (IHC) using anti-collagen I (1:1,000; Abcam; cat. no. 34710). The infiltration of inflammatory cells was detected by IHC with CD4 (1:1,000; Abcam; cat. no. 183685), CD8 [1:1,000; Cell
Signaling Technology, Inc. (CST); cat. no. 98941] and F4/80 (1:1,000; CST; cat. no. 70076) antibodies. Next, 5 different fields were randomly selected and imaged under an upright light microscope (Leica DM750). The average areas of tissue fibrosis, relative size of cells that are positive in IHC were measured using ImageJ software (National Institutes of Health)

Western blotting. Mouse heart tissues and primary cells were collected and protein concentrations were quantified using a bicinchoninic acid protein assay kit (Thermo Fisher). Equivalent amounts of proteins (30-50 $\mu \mathrm{g}$ ) were loaded on SDS-polyacrylamide gels (10-15\%) and performed as previously described (18). Proteins were transferred onto polyvinylidene fluoride membranes and blocked (RT, $30 \mathrm{~min}$ ) with $5 \%$ nonfat dry milk. The membranes were then incubated with the following primary antibodies: Total p38-MAPK (CST; cat. no. 8690], phospho-p38-MAPK (p-p38; CST; cat. no. 4511). Tubulin (CST; cat. no. 2146) was used as the control. Following washing with PBS with $0.1 \%$ Tween-20, the membranes were incubated with the corresponding secondary antibodies (1:5,000; Anti-rabbit IgG; CST; cat. no. 7074). The bands were visualized using an enhanced chemiluminescence system (Minichemi ${ }^{\mathrm{TM}}$; Beijing Saiz Science \& Technology Co., Ltd.). The intensity of each protein band was quantified using ImageJ software.

Reverse transcription-quantitative polymerase chain reaction ( $R T-q P C R)$. Total RNA was extracted from mouse hearts or primary cells using TRIzol reagent (Thermo Fisher Scientific, Inc.). For mRNA detection in mouse hearts, infarcted tissues plus border zone tissues in MI/R mice and the tissues of similar region in sham mice were used. Then the RNA concentration was quantified using a NanoDrop 2000 (Thermo Fisher Scientific, Inc.) and cDNA was obtained using the PrimerScript RT reagent kit at $37^{\circ} \mathrm{C}$ for $15 \mathrm{~min}$, and $85^{\circ} \mathrm{C}$ for $5 \mathrm{sec}$ (Takara Bio, Inc.). The mRNA levels were detected by RT-qPCR with a SYBR Green Master Mix kit (Takara Bio, Inc.) using real-time PCR systems (Thermo Fisher Scientific, 
Table I. The primer sequences information.

\begin{tabular}{lll}
\hline Gene & \multicolumn{1}{c}{ Forward primer (5'-3') } & \multicolumn{1}{c}{ Reverse primer (5'-3') } \\
\hline m- $\beta$-actin & GGCTGTATTCCCCTCCATCG & CCAGTTGGTAACAATGCCATGT \\
m-ANP & GCTTCCAGGCCATATTGGAG & GGGGGCATGACCTCATCTT \\
m-BNP & AGTCCTTCGGTCTCAAGGCA & CCGATCCGGTCTATCTTGTGC \\
m- $\alpha$-SMA & GTCCCAGACATCAGGGAGTAA & TCGGATACTTCAGCGTCAGGA \\
m-collagen-I & GCTCCTCTTAGGGGCCACT & ATTGGGGACCCTTAGGCCAT \\
m-IL-1 $\beta$ & CGAGGCTAATAGGCTCATCT & GTTTGGAAGCAGCCCTTCAT \\
m-IL-6 & TGATGCACTTGCAGAAAACA & ACCAGAGGAAATTTCAATAGGC \\
m-Arg1 & CAGAAGAATGGAAGAGTCAG & CAGATATGCAGGGAGTCACC \\
m-CD206 & AACAAGAATGGTGGGCAGTC & AACTCCTCGTCCGTCTGTC \\
m-iNOS & CACCAAGCTGAACTTGAGCGA & CCATAGGAAAAGACTGCACCGA \\
m-TNF- $\alpha$ & GCCAACGGCATGGATCTC & GCAGCCTTGTCCCTTGAAGAG \\
m-Ang II & CCAGGCCCGTTGTTCTTGAT & GGAAGGGAGACTTGCTCATTC \\
r- $\beta$-actin & CGTGCGTGACATTAAAGAG & TTGCCGATAGTGATGACCT \\
r-GAPDH & CGGCAAGTTCAACGGCACAG & CGCCAGTAGACTCCACGACAT \\
r-collagen-I & GGAGAGAGTGCCAACTCCAG & GTGCTTTGGAAAATGGTGCT \\
r-TNF- $\alpha$ & CTGTGCCTCAGCCTCTTCTC & ACTGATGAGAGGGAGCCCAT \\
r-ANP & GGTCCTTCGGATGCAGTATT & CCACTTGAGCAGCATTGTGT \\
r-IL-1 $\beta$ & ACGGGTTCCATGGTGAAGT & CCTCTCAAGCAGAGCACAGA \\
r-IL-6 & CCAGCCAGTTGCCTTCT & GTCCTTAGCCACTCCTTCT \\
r-Ang II & TTGGATAAAGAACCCGCCTC & AAAGGGTAGACAGCTTGGC \\
\hline
\end{tabular}

IL, interleukin; TNF, tumor necrosis factor; CD, cluster of differentiation; Ang II, angiotensin II; iNOS, inducible nitric oxide synthase; ANP, atrionatriuretic protein; BNP, brain natriuretic protein; SMA, smooth muscle actin.

Inc.). The thermocycling conditions were as follows: $96^{\circ} \mathrm{C}$ for $30 \mathrm{sec}, 57^{\circ} \mathrm{C}$ for $30 \mathrm{sec}$ and $72^{\circ} \mathrm{C}$ for $30 \mathrm{sec}$. The mRNA expression level was calculated using the $2^{-\Delta \Delta \mathrm{Cq}}$ method (18). The $\beta$-actin and GAPDH genes were used as the control. The primer sets used to identify the gene are given in Table I.

Statistical analysis. Results were analyzed using GraphPad Prism 5.0 analysis software (GraphPad Software, Inc.) and are expressed as the mean \pm standard error of the mean of 3 independent experiments. In the present study, an unpaired two-tailed student's t-test was used for comparisons between different groups. One-way analysis of variance with a Bonferroni post hoc test was used for multiple comparisons. $\mathrm{P}<0.05$ was considered to indicate a statistically significant difference and $\mathrm{P}<0.01$, a highly statistically significant difference. The survival curve was estimated by the Log-rank (Mantel-Cox) Test.

\section{Results}

Safety of VCP979 treatment in mice and cultured cells. To ensure the safety of this newly developed small-molecule compound, VCP979, the body weight of mice that received VCP979 (50 mg/kg/day, i.p.) or control solvent for 14 consecutive days was first evaluated. As shown in Fig. 2A, there was no significant difference in body weight between the two sham groups on days $0,7,14,21$ and 28 after surgery. H\&E staining results showed that the administration of VCP979 had no obvious impact on the pathology of multiple murine organs, including the lung, liver, kidney, spleen and heart (Fig. 2B).
Moreover, AST, ALT and LDH levels in serum were measured in sham groups and no difference was observed after VCP979 injection (Fig. 2C).

In addition, to clarify whether VCP979 could be toxic to primary cells, the percentages of LDH leakage in the NCFs and NCMs treated with or without VCP979 for $24 \mathrm{~h}$ were assessed. Fig. 2D and $\mathrm{E}$ show that different concentrations of VCP979 $(0.1,1,3$ and $9 \mu \mathrm{M})$ do not significantly affect the percentages of LDH leakage in NCFs and NCMs. These results indicated no significant toxic effects of VCP979 at the present concentrations and duration.

VCP979 administration improves cardiac function in murine MI/R injury models. Cardiac function was examined by $\mathrm{M}$-mode echocardiography on day 1 before and days 1 , 7, 14 and 28 after surgery (Fig. 3A). As compared with the MI/R group, the administration of VCP979 significantly increased both LVEF $(28.1908 \pm 4.1065$ vs. $40.5348 \pm 3.4986)$ and LVFS $(14.2999 \pm 2.1439$ vs. $21.372 \pm 2.3741)$ on day 14 $(\mathrm{P}<0.05)$, but decreased both LV Vol; d/s $(99.3371 \pm 6.8049$ vs. $69.3894 \pm 4.1022 / 51.8582 \pm 2.0042$ vs. $37.1314 \pm 2.6628)$ and LVID; d/s (4.6353 \pm 0.1338 vs. $3.8792 \pm 0.1285 / 3.8035 \pm 0.2779$ vs. $2.8924 \pm 0.2081$ ) on day 28 (Fig. 3A). These results demonstrated that VCP979 could improve ventricular regional and global function (LVEF and LVFS) and inhibit the progression of ventricular remodeling (LV Vol; $d / s$ and LVID; $d / s$ ). In addition, there was no significant difference in the survival rate between the sham and MI/R groups injected with N.S or VCP979 during the 28 days of observation (Fig. 3C). 

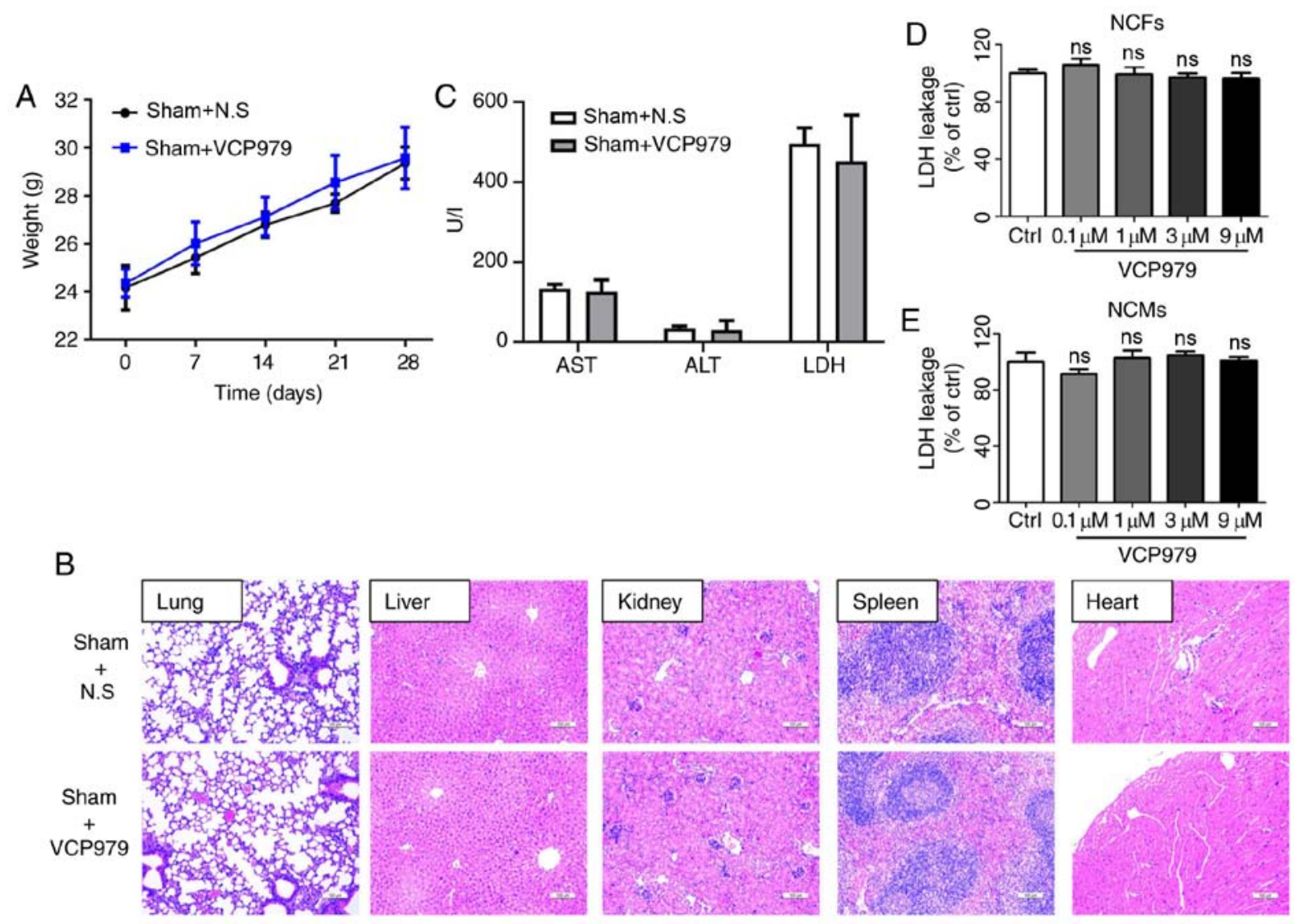

Figure 2. Safety of VCP979 treatment in mice and cultured cells. (A) The body weight of mice in the sham groups was recorded on days $0,7,14,21$ and 28 following surgery ( $\mathrm{n}=6$ per group). (B) Representative hematoxylin and eosin-stained histological sections of the lung, liver, kidney, spleen and heart of mice after 28 days. Scale bar=100 $\mu \mathrm{m}$. (C) The AST, ALT and LDH levels in serum were measured in sham groups. LDH leakage percent of the (D) NCFs and (E) NCMs following treatment with or without different concentrations of VCP979 (0.1, 1, 3 and $9 \mu \mathrm{M})$ for $24 \mathrm{~h}$. NCF, neonatal cardiac fibroblast; NCM, neonatal cardiac myocyte; LDH, lactate dehydrogenase; AST, aspartate transaminase; ALT, alanine aminotransferase.

VCP979 administration attenuates myocardial fibrosis following MI/R injury. Next, myocardial fibrosis was analyzed by Masson's Trichrome staining and IHC. Fig. 4A and C showed that $\mathrm{MI} / \mathrm{R}$ surgery significantly increased the average area of myocardial fibrosis on day $28(\mathrm{P}<0.001)$, compared with the sham mice $(0.4283 \pm 0.1253$ vs. $2.3253 \pm 0.1181)$ and the administration of VCP979 greatly reduced the fibrosis area, as compared with the N.S-treated MI/R group (2.3253 \pm 0.1181 vs. $1.245 \pm 0.0801)$. Furthermore, treatment with VCP979 attenuated collagen I expression in MI/R hearts (12.247 \pm 0.5022 vs. $9.805 \pm 0.4045$, Fig. $4 \mathrm{~B}$ and D). As shown by Fig. 4E, mRNA levels of $\alpha$-smooth muscle actin ( $\alpha$-SMA), collagen I and Ang II were significantly decreased in the MI/R+VCP979 group, as compared with the MI/R+N.S group on day $28(1.5504 \pm 0.0923$ vs. $1.257 \pm 0.0514,2.9636 \pm 0.225$ vs. $1.5357 \pm 0.2741$ and $2.9806 \pm 0.1888$ vs. $1.3883 \pm 0.0974$, respectively; $\mathrm{P}<0.05)$. These findings suggested that VCP979 plays an important role in attenuating myocardial fibrosis in murine $\mathrm{MI} / \mathrm{R}$ injury models.

VCP979 treatment alleviates pathological hypertrophy of hearts subjected to MI/R injury. To evaluate the role of VCP979 on pathological hypertrophy, the CSAs were detected by WGA staining. Fig. 5A and B showed that the average cell size in the MI/R+VCP979 group was significantly decreased compared with in the MI/R+N.S group $(541.3636 \pm 54.7254$ vs. 305.9167 \pm 28.6484 ; $\mathrm{P}<0.001)$. In addition, the continuous 2-weeks VCP979 injection significantly reduced the ratio of heart weight ( $\mathrm{g}$ ) to body weight $(\mathrm{g})$, as compared with N.S injection in $\mathrm{MI} / \mathrm{R}$ mice $(0.469 \pm 0.0037$ vs. $0.4417 \pm 0.0064 ; \mathrm{P}<0.05$; Fig. 5C). Furthermore, RT-qPCR analysis of the infarcted area and border zone of left ventricle demonstrated that VCP979 treatment significantly decreased the mRNA levels of atrial natriuretic peptide (ANP) and brain natriuretic peptide (BNP) on day 28, compared with the N.S-treated MI/R group $(24.9505 \pm 1.2631$ vs. $18.8687 \pm 0.3303$ and $2.2903 \pm 0.0402$ vs. $1.482 \pm 0.0653$, respectively; $\mathrm{P}<0.01$; Fig. 5D). This phenomenon suggested that VCP979 plays a protective role in MI/R injury induced pathological hypertrophy.

VCP979 treatment prevents inflammatory cell infiltration and suppresses the p38-MAPK signaling pathway in $M I / R$ hearts. Pro-inflammatory cell infiltration has been reported to be closely associated with heart injury $(14,19)$. In order to verify the potential mechanism through which the administration of VCP979 protects heart function following $\mathrm{MI} / \mathrm{R}$ injury, the infiltration of inflammatory cells was detected by IHC and the results revealed fewer numbers of cluster of differentiation (CD) $4^{+}, \mathrm{CD}^{+}$and $\mathrm{F} 4 / 80^{+}$inflammatory cells in hearts of MI/R+VCP979 group compared with those from the MI/R+N.S group on day 14 post-MI/R injury $(0.7508 \pm 0.0891$ vs. $0.2296 \pm 0.0555,0.5315 \pm 0.0819$ 

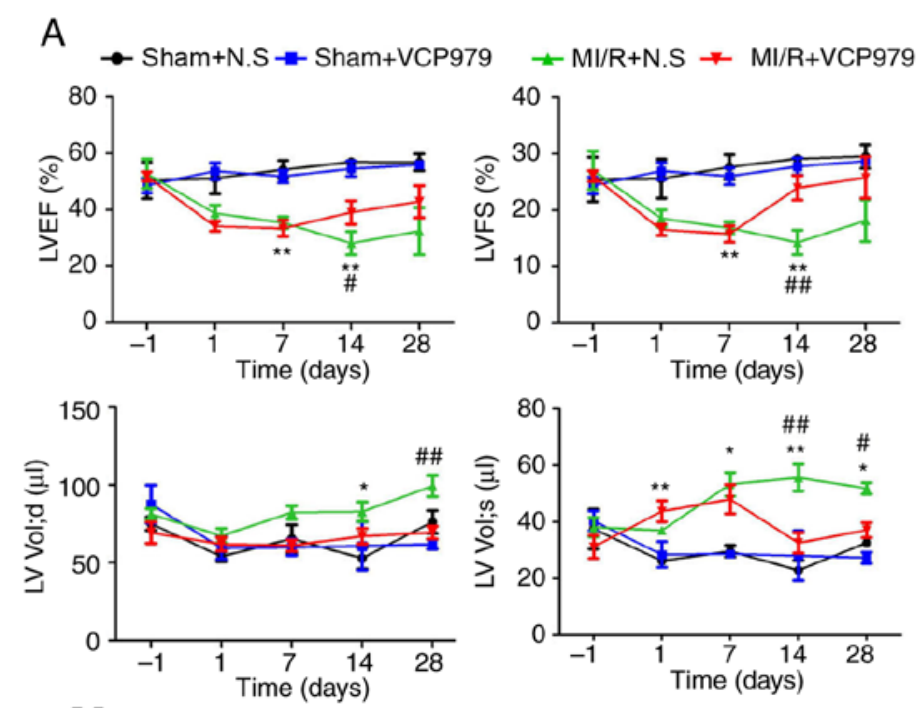
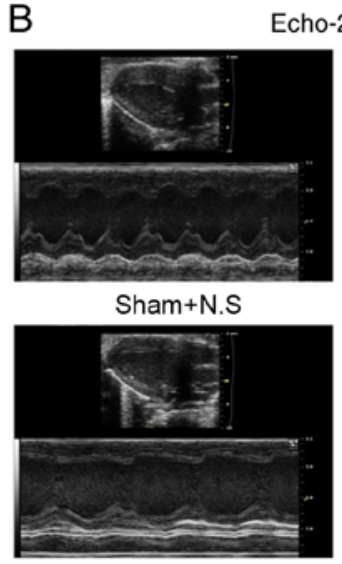

MI/R+N.S

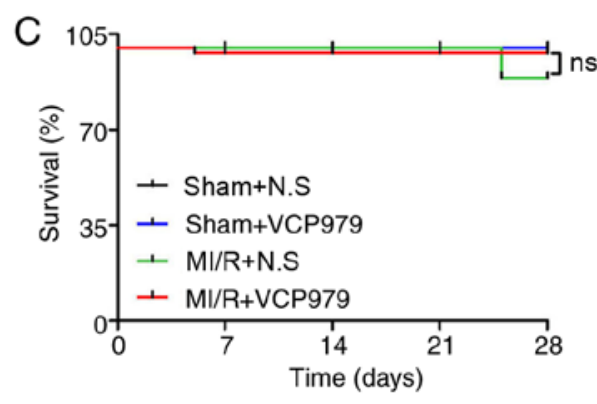

Figure 3. VCP979 administration improved cardiac function in mice post-MI/R injury. (A) Cardiac function was detected on days 1 before and 1, 7, 14 and 28 after surgery ( $\mathrm{n}=6$ per group). (B) Representative images of M-mode echocardiography from the sham+N.S, sham+VCP979, MI/R+N.S and MI/R+VCP979 groups on day 28. (C) The survival curves of the sham and MI/R groups injected with N.S or VCP979 were observed for 28 days ( $\mathrm{n}=12$ per group). Results are expressed as the mean \pm standard error of the mean. ${ }^{*} \mathrm{P}<0.05$ and ${ }^{* *} \mathrm{P}<0.01$, sham $+\mathrm{N} . S$ vs. MI/R+N.S group. ${ }^{*} \mathrm{P}<0.05$ and ${ }^{\# \#} \mathrm{P}<0.01, \mathrm{MI} / \mathrm{R}+\mathrm{N} . \mathrm{S}$ vs. MI/R+VCP979. N.S, normal saline; MI/R, myocardial ischemia/reperfusion; LVEF, left ventricular ejection fraction; LV vol, left ventricular volume; LVFS, left ventricular fractional shortening; LVIDd/s, left-ventricular internal dimension; diastolic/systolic.

vs. $0.2414 \pm 0.0198$ and $2.8303 \pm 0.3082$ vs. $1.7483 \pm 0.1626$, respectively; Fig. 6A and B). Next, the mRNA expression of several inflammatory cytokines and macrophage polarization-related factors in murine heart tissues was assessed on day 14. Fig. 6C showed that, as compared with the MI/R group, VCP979 treatment significantly increased the levels of arginase 1 (Arg1) and CD206 (1.0092 \pm 0.0937 vs. $1.6763 \pm 0.0677$ and $1.0021 \pm 0.0467$ vs. $1.3567 \pm 0.0072$, respectively; $\mathrm{P}<0.01$ ), while it decreased inducible nitric oxide synthase (iNOS), IL-6 and TNF- $\alpha(1.0032 \pm 0.0565$ vs. $0.4094 \pm 0.0534,1.0127 \pm 0.1089$ vs. $0.3444 \pm 0.0311$ and $1.1481 \pm 0.0655$ vs. $0.8184 \pm 0.0037$, respectively) mRNA levels on day 14 after surgery.

Next whether VCP979 could suppress the p38-MAPK signaling pathway in vivo was explored. Fig. 6D and E showed that the administration of VCP979 significantly decreased the ratio of p-p38/p38 in MI/R hearts compared with the N.S treatment MI/R group (1.0005 \pm 0.1884 vs. $0.1236 \pm 0.0164$; $\mathrm{P}<0.05)$.

Addition of VCP979 inhibits the Ang II-induced proliferation and collagen synthesis of NCFs and hypertrophy of NCMs, and suppresses the p38-MAPK signaling pathway. To explored whether VCP979 exerts its functions on fibroblasts and cardiomyocytes directly, NCFs and NCMs were isolated and the effect of VCP979 on cells exposed to Ang II $(200 \mathrm{nM})$ was investigated. As shown in Fig. 7A, $24 \mathrm{~h}$ stimulation with Ang II significantly increased the proliferation of NCFs $(100.1229 \pm 1.2103$ vs. $107.7961 \pm 2.902 ; \mathrm{P}<0.05)$ and high concentrations $(1,3$ and $9 \mu \mathrm{M})$ of VCP979 markedly reversed this elevation $(96.9753 \pm 0.8734,93.0633 \pm 0.7293$ and $91.28 \pm 0.4052$, respectively; Fig. 7A). Meanwhile, the collagen I mRNA level in NCFs was significantly reduced following treatment with high concentrations $(1,3$ and $9 \mu \mathrm{M})$ of VCP979 for $24 \mathrm{~h}(1.1763 \pm 0.013$ vs. $1.0471 \pm 0.0051$, $0.8838 \pm 0.0096,0.8916 \pm 0.0028$, respectively; $\mathrm{P}<0.05$; Fig. 7B). Next, VCP979 treatment was shown to significantly decrease the ANP mRNA level in Ang II-induced NCMs at different concentrations $(0.1,1,3$ and $9 \mu \mathrm{M} ; 38.8748 \pm 0.4596$ vs. $26.2532 \pm 1.3328,20.0562 \pm 0.8087,19.568 \pm 1.0699$ and $15.474 \pm 1.8371$, respectively; $\mathrm{P}<0.05$; Fig. 7C). Phalloidin staining was performed to evaluate the role of VCP979 against Ang II-stimulated cardiac hypertrophy. As shown in Fig. 7D and E, Ang II notably increased the average cell size of NCMs $(4483.127 \pm 369.8357$ vs. $7986.913 \pm 236.3841)$, which could be partially reversed by treatment with VCP979 ( $9 \mu \mathrm{M}, 24 \mathrm{~h}, 6680.613 \pm 385.5386)$. Furthermore, the present results revealed that the addition of VCP979 $(9 \mu \mathrm{M}, 24 \mathrm{~h})$ can decrease the ratio of p-p38/p38 in NCFs exposed to Ang II in vitro $(2.9061 \pm 0.1981$ vs. $0.1473 \pm 0.0602$, Fig. $7 \mathrm{~F}$ and $\mathrm{G})$. In addition, VCP979 treatment can significantly decreased the expression of IL-1 $\beta$, IL- 6 and TNF- $\alpha$ mRNA levels in NCFs $(3.4058 \pm 0.2912$ vs. $1.8504 \pm 0.2208,7.5461 \pm 0.9675$ vs. $1.5955 \pm 0.2052$ and $2.3879 \pm 0.1921$ vs. $0.6044 \pm 0.0533$, respectively; $\mathrm{P}<0.05$; Fig. $7 \mathrm{H})$ and $\mathrm{NCMs}(2.687 \pm 0.2218$ 
A

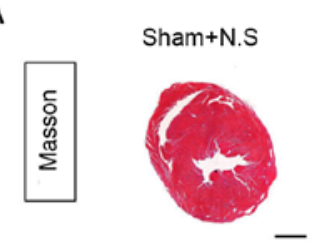

B

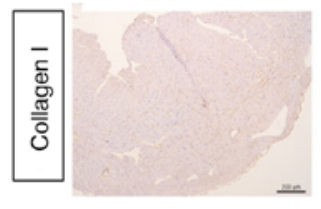

E

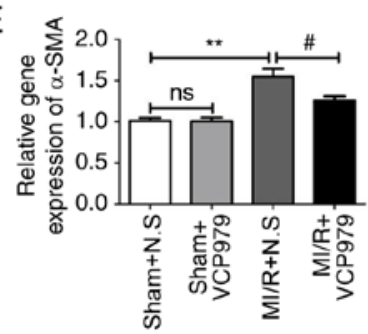

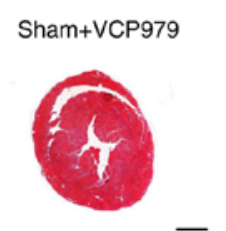

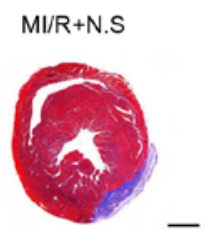

MI/R+VCP979

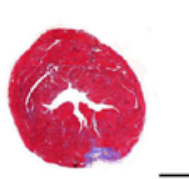

C
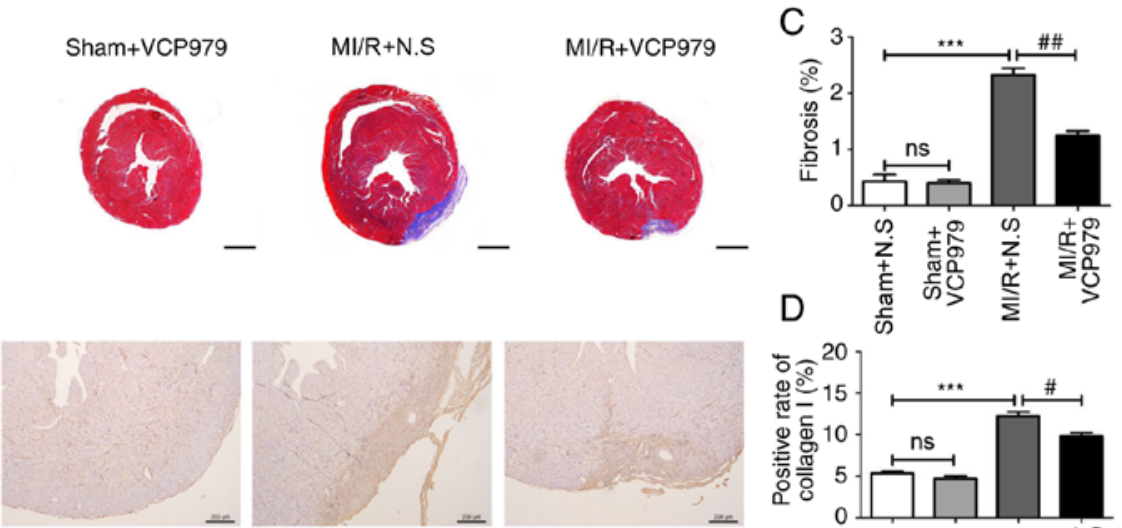
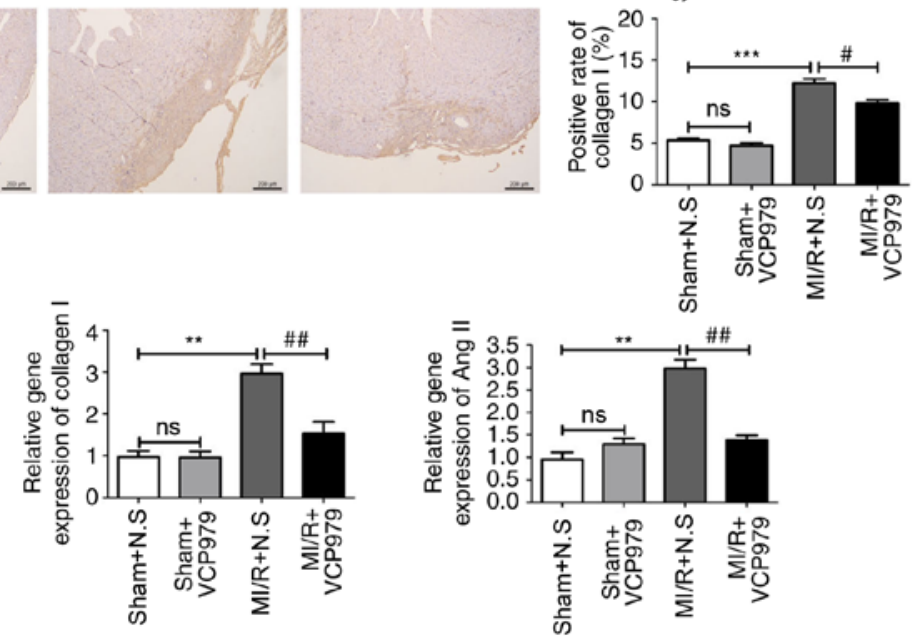

Figure 4. VCP979 administration of attenuated myocardial fibrosis following MI/R injury. (A) Representative Masson's Trichrome-stained histological sections of hearts from the sham+N.S, sham+VCP979, MI/R+N.S and MI/R+VCP979 groups. Scale bar=1 mm. (B) Representative collagen I staining pictures. Scale bar $=200 \mu \mathrm{m}$. (C) The fibrosis area of heart tissues and the (D) positive rate of collagen I were measured using ImageJ software. (E) mRNA levels of $\alpha$-SMA, collagen I and Ang II in heart tissues of different groups. Results are expressed as the mean \pm standard error of the mean. ${ }^{* *} \mathrm{P}<0.01$ and ${ }^{* * *} \mathrm{P}<0.001$, sham+N.S vs. MI/R+N.S. " $\mathrm{P}<0.05$ and ${ }^{\# \#} \mathrm{P}<0.01, \mathrm{MI} / \mathrm{R}+\mathrm{N} . \mathrm{S}$ vs. MI/R+VCP979. SMA, smooth muscle actin; MI/R, myocardial ischaemia/reperfusion; N.S, normal saline; Ang II, angiotensin II; ns, not significant.
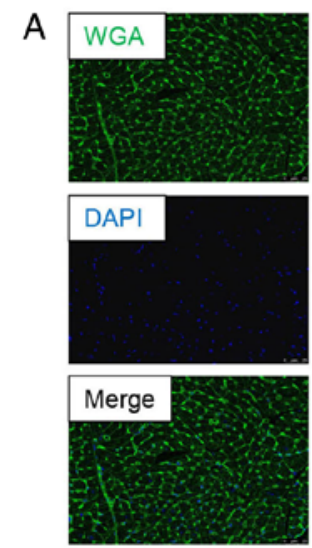

Sham+N.S
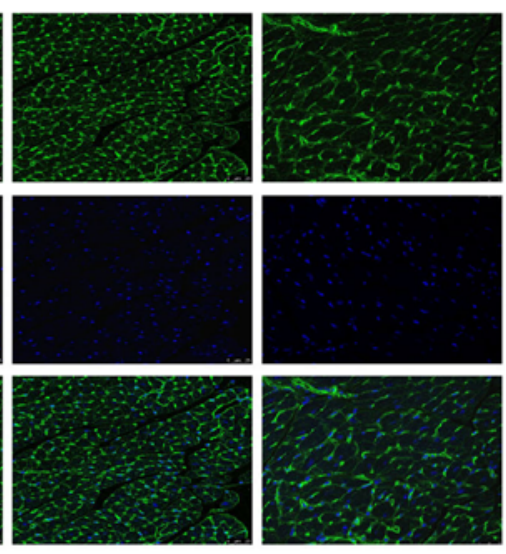

Sham+VCP979

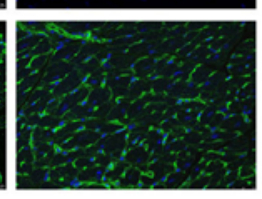

$\mathrm{Ml} / \mathrm{R}+\mathrm{N} . \mathrm{S}$

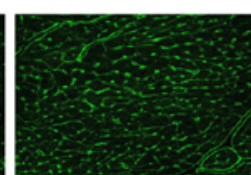

B

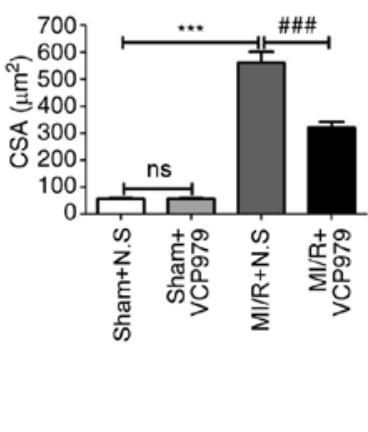

$\mathrm{Ml} / \mathrm{R}+\mathrm{VCP} 979$
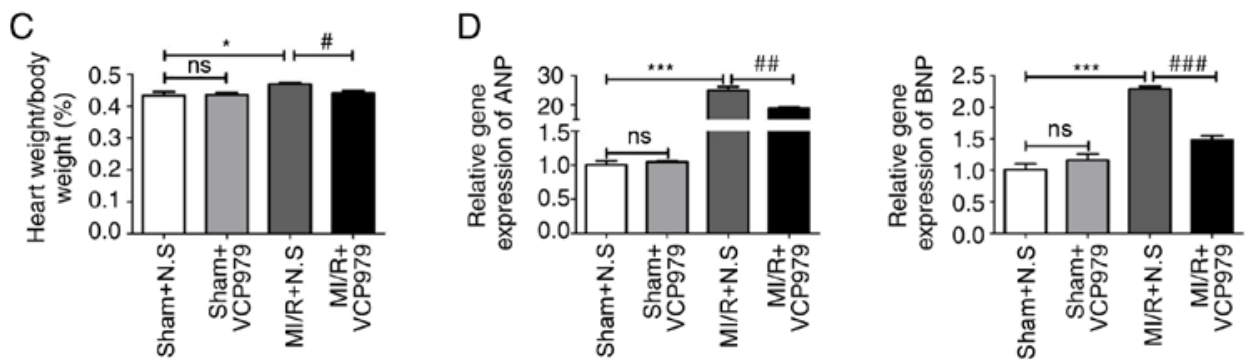

Figure 5. VCP979 treatment can alleviate pathological hypertrophy of the heart after day 28 in vivo. (A) Representative WGA immunofluorescence-stained sections. Green, WGA; Blue, nucleus. Scale bar=25 $\mu \mathrm{m}$. (B) The cross-sectional areas of the heart sections were estimated using ImageJ software. (C) The ratio of heart weight (g) to body weight (g) post-MI/R injury on day 28. (D) mRNA expression of ANP and BNP in hearts. Results are expressed as the mean \pm standard error of the mean. ${ }^{*} \mathrm{P}<0.05$ and ${ }^{* * * *} \mathrm{P}<0.001$, sham+N.S vs. MI/R+N.S. ${ }^{\# P}<0.05,{ }^{\#} \mathrm{P}<0.01$ and ${ }^{\# \#} \mathrm{P}<0.001$. MI/R+N.S vs. MI/R+VCP979. WGA, wheat germ agglutinin; ANP, atrial natriuretic peptide; BNP, brain natriuretic peptide; MI/R, myocardial ischemia/reperfusion; N.S, normal saline; ns, not significant. 
A
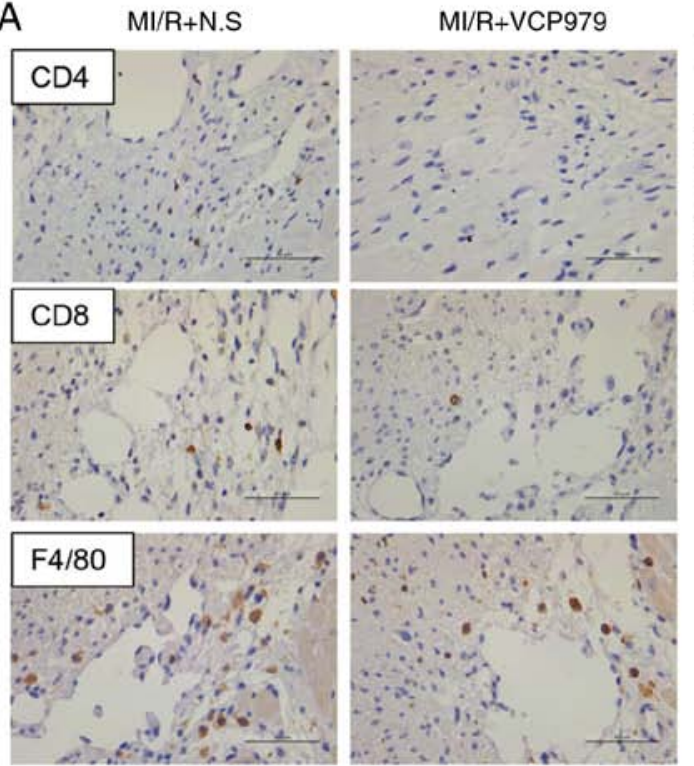

D

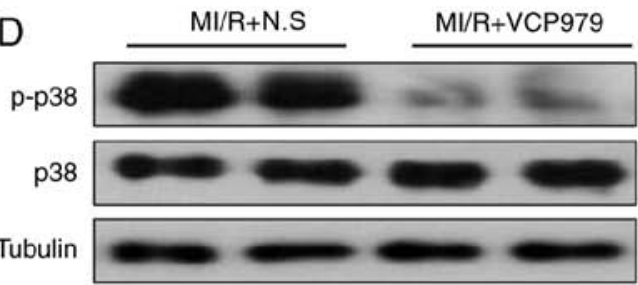

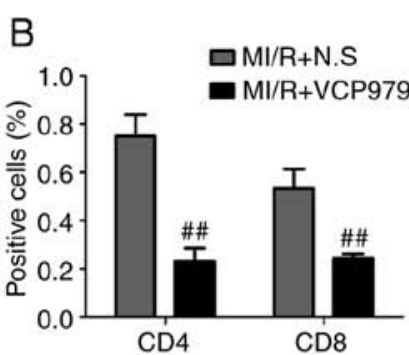
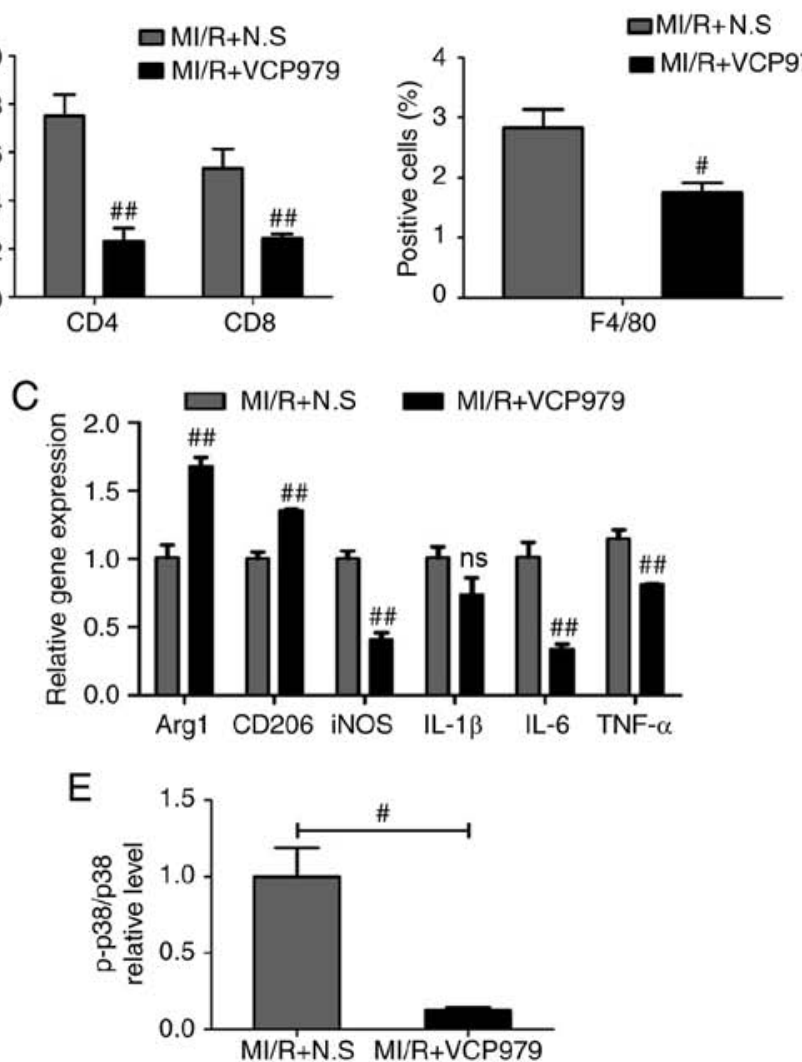

Figure 6. VCP979 treatment prevents inflammatory cell infiltration and suppresses the p38-MAPK signaling pathway on day 14. (A) Representative CD4, CD8 and F4/80 staining pictures. Scale bar=50 $\mu \mathrm{m}$. (B) The relative positive cells of the sections were determined by ImageJ software. (C) Reverse transcription-quantitative PCR analysis of mRNA levels of Arg1, CD206, iNOS, IL-1 $\beta$, IL-6 and TNF- $\alpha$ in the heart tissues. (D) Western blotting analysis and (E) quantitative analysis of p-p38 and p38 protein levels in heart tissues from the MI/R+N.S and MI/R+VCP979. Results are shown as mean \pm standard error of the mean. ${ }^{\#} \mathrm{P}<0.05$ and ${ }^{\# \#} \mathrm{P}<0.01, \mathrm{MI} / \mathrm{R}+\mathrm{N} . \mathrm{S}$ vs. MI/R+VCP979 group. MAPK, mitogen-activated protein kinase; iNOS, inducible nitric oxide synthase; p-, phosphorylated-; IL, interleukin; TNF, tumor necrosis factor; CD, cluster of differentiation; MI/R, myocardial ischemia/reperfusion; N.S, normal saline; ns, not significant; Arg1, arginase 1.

vs. $2.4503 \pm 0.6911,3.1266 \pm 0.3756$ vs. $0.3447 \pm 0.0316$ and $1.4729 \pm 0.0503$ vs. $0.7569 \pm 0.0552$, respectively; Fig. 7I), compared with the Ang II group.

\section{Discussion}

Early coronary reperfusion can limit the infarct size and improve prognosis, while persistent reperfusion can itself induce cardiomyocyte damage. Existing pharmacological treatment is not entirely capable of preventing the progression of ventricular remodeling in $\mathrm{MI} / \mathrm{R}$ injury and new drugs for ischemic heart disease are still being developed (20). In the present study, it was found that the administration of VCP979 attenuated MI/R-induced cardiac dysfunction, markedly alleviated myocardial fibrosis and pathological hypertrophy, and had an anti-inflammatory effect in murine MI/R injury models. In vitro, VCP979 treatment inhibited Ang II-induced proliferation and collagen synthesis in NCFs and hypertrophy in NCMs. VCP979 is a selective inhibitor for p38-MAPK $\alpha$ and is a much poor inhibitor of $\mathrm{p} 38$-MAPK $\beta$. It does not inhibit the $\mathrm{p} 38-\mathrm{MAPK} \gamma$ or $\delta$ isoform. A previous study revealed that p38-MAPK $\alpha$ is most readily detected in cardiac myocytes or whole hearts with little or no p38-MAPK $\beta$ (21). Furthermore, VCP979 treatment inhibited the p38-MAPK signaling pathway in murine hearts subjected to $\mathrm{MI} / \mathrm{R}$ injury as well as Ang II-induced NCFs collagen synthesis. Therefore, these findings provided the first evidence that a novel small-molecule compound, VCP979, may be an effective strategy for the protection of the heart from MI/R injury.

First, the safety of this newly developed small-molecule compound VCP979 was verified in vivo and in vitro. The results confirmed that different concentrations of VCP979 (50 $\mathrm{mg} / \mathrm{kg} / \mathrm{day}$ in vivo and 0.01-9 $\mu \mathrm{M}$ in vitro, the dose chosen is based on preliminary PK data) caused no pathological damage on murine organs and primary cells, suggesting the safety of VCP979 treatment in murine models and cultured cells. As it is in the early development process, the pharmacokinetics of the compound in vivo is not fully clear and needs further investigation.

Ventricular remodeling triggered by MI/R injury is a progressive process that eventually leads to heart failure (3). Cardiac fibroblasts play vital and dynamic roles in regulating cardiac function (4). Under physiological conditions, cardiac fibroblasts supply a scaffold for cardiomyocytes and ensure cardiac pump function (4). By contrast, cardiac fibroblasts expand following injury,(22) and participate in the pathogenesis of adverse post-infarction remodeling (23). Cardiac hypertrophy is another key pathological change that follows MI/R 

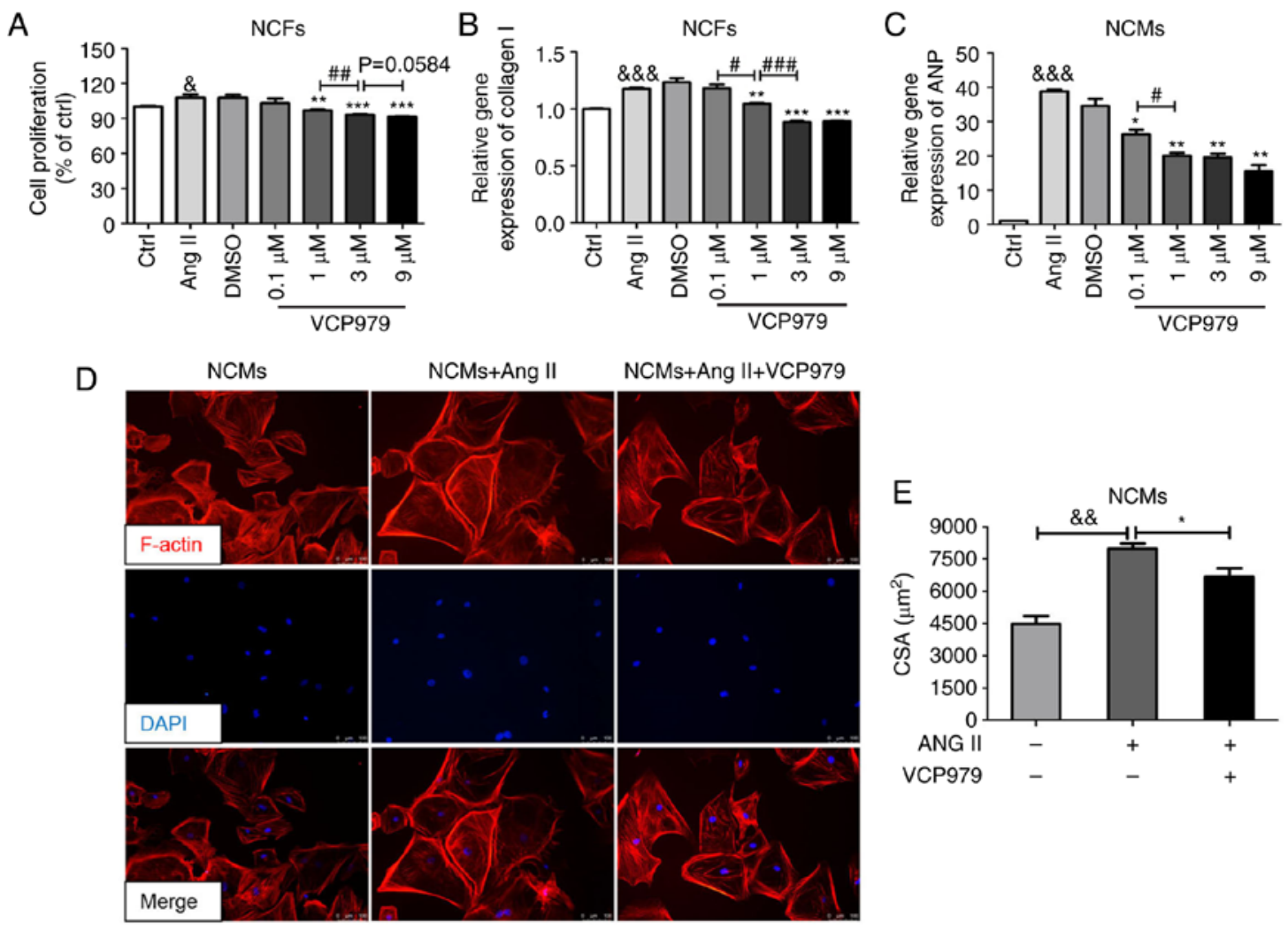

NCMs+Ang II+VCP979

$\mathrm{F}$

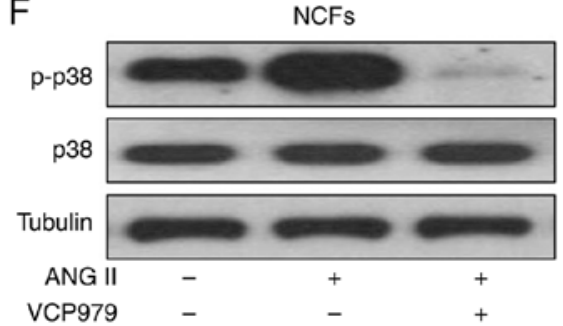

$\mathrm{H}$

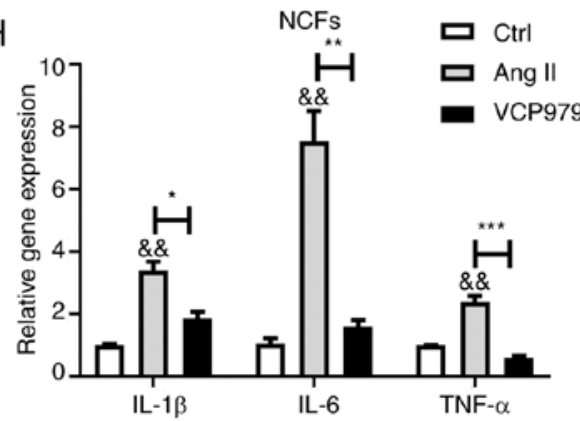

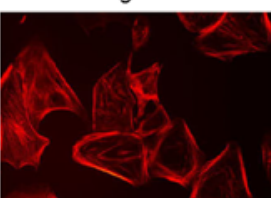

E

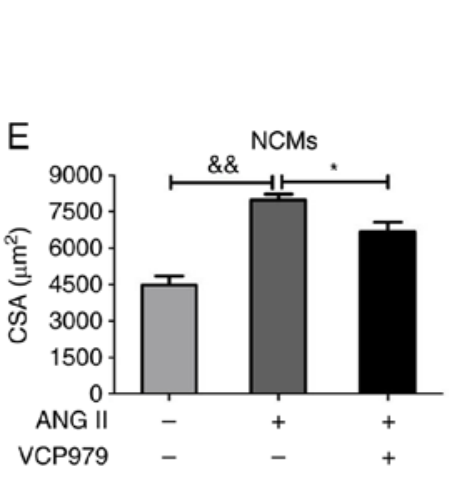

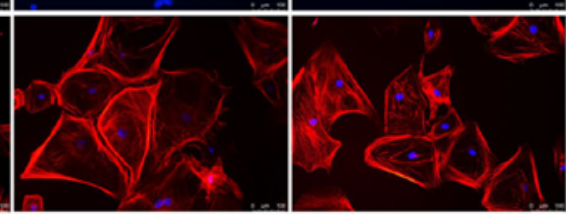

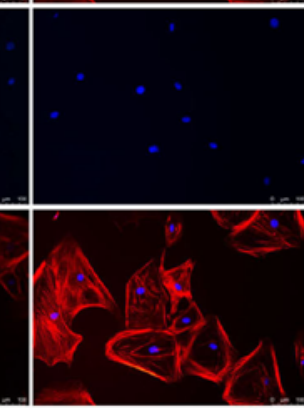

G

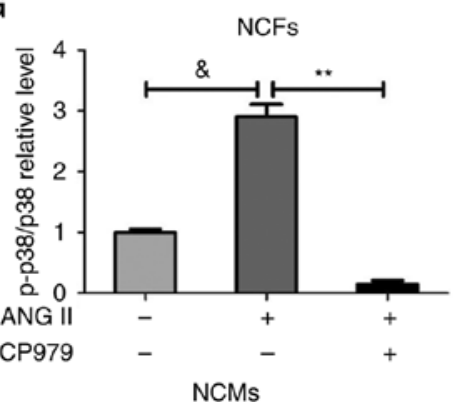

।

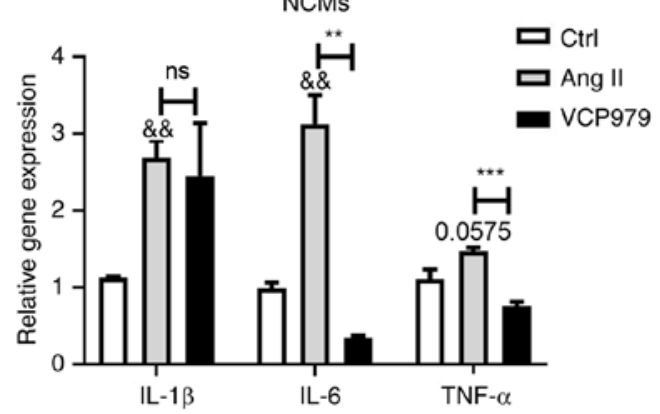

Figure 7. Addition of VCP979 inhibits the Ang II-induced proliferation and collagen synthesis of NCFs and hypertrophy of NCMs and suppresses the p38-MAPK signaling pathway in vitro. (A) Ang II-induced proliferation percentage in NCFs following treatment with different concentrations of VCP979. (B) Collagen I mRNA level in NCFs and (C) ANP mRNA level in NCMs following treatment with VCP979 for 24 h. (D) Representative Phalloidin immunofluorescence staining pictures of NCMs. Phalloidin is highly integrated with F-actin. Red, F-actin; Blue, nucleus. Scale bar=100 $\mu \mathrm{m}$. (E) The CSAs of the NCMs were measured using ImageJ software. (F) Western blotting and (G) analysis of p-p38 proteins in Ang II-induced NCFs, with or without the addition of VCP979. The mRNA expression of IL-1 $\beta$, IL-6 and TNF- $\alpha$ in (H) NCFs and (I) NCMs. Results are presented as the mean \pm standard error of the mean. ${ }^{\&} \mathrm{P}<0.05$, ${ }^{\& \&} \mathrm{P}<0.01$ and ${ }^{\& \& \&} \mathrm{P}<0.001$, control vs. Ang II-induced group. ${ }^{*} \mathrm{P}<0.05,{ }^{* *} \mathrm{P}<0.01$ and ${ }^{* * * *} \mathrm{P}<0.001$, Ang II-induced vs. VCP979 treatment group. ${ }^{\#} \mathrm{P}<0.05$, ${ }^{\# \#} \mathrm{P}<0.01$ and ${ }^{\# \# \#} \mathrm{P}<0.001$, significant differences between various concentrations of VCP979 in (A-C). NCFs, neonatal cardiac fibroblasts; NCMs, neonatal cardiac myocytes; p-, phosphorylated-; MAPK, mitogen-activated protein kinase; ANP, atrial natriuretic peptide; CSAs, myocyte cross-sectional areas; IL, interleukin; TNF, tumor necrosis factor; CD, cluster of differentiation; Ang II, angiotensin.

injury and contributes to cardiac dysfunction (24). Persistent pathological cardiac hypertrophy leads to increased physical, oxidative and nitrosative stress at the cellular level and further alters the shape, size and function of the myocardium (25).
Therefore, targeting pathological myocardial fibrosis and hypertrophy may be a promising therapeutic strategy for ventricular remodeling (25). However, few effective drugs can prevent or reverse the process of ventricular remodeling (25). 
It has been previously reported that the long-term daily treatment of rats with the p38-MAPK signaling pathway inhibitor RWJ67657 can reduce pathological myocardial fibrosis and hypertrophy and improve cardiac function (LVFS) following MI (26). Similarly, the present results showed a reduction in pathological myocardial fibrosis and hypertrophy accompanied by improved cardiac function following 2 weeks of continuous VCP979 administration, which indicated that VCP979 may be useful for alleviating ventricle remodeling following MI/R injury.

Reperfusion damage accelerates the infiltration of inflammatory leukocytes into the injured myocardium (27). Inflammatory responses activated by MI/R injury contribute to collagen deposition and cardiac hypertrophy, but reduce left ventricular compliance (19). Cardiac inflammation is characterized by the recruitment and activation of immune cells mainly neutrophils, macrophages and lymphocytes at different stages post MI/R injury (3). T cell-mediated chronic inflammation has been confirmed as a major player in the etiology and development of ventricular remodeling post-MI/R injury (28). Subsequent studies have shown the role of $\mathrm{CD}^{+} \mathrm{T}$-cells in the pathology and healing of the heart following MI or MI/R injury (29-31). Recent results showed an increase in CD4 ${ }^{+} \mathrm{T}$ cells accompanied by high levels of $\mathrm{CD}^{+} \mathrm{T}$ cell-associated inflammatory factors in $\mathrm{MI} / \mathrm{R}$ myocardia on days 7 and 14, as compared with the sham group (14). The present study revealed that VCP979 inhibited the infiltration of $\mathrm{CD}^{+}$cells in injured hearts on day 14 following MI/R injury. As for $\mathrm{CD}^{+} \mathrm{T}$ cells, little is known about their role in MI/R injury. A subset of $\mathrm{CD}^{+}$ cells expressing AT2R was shown to have no cytotoxic activity, suggesting a potential cardioprotective role in rat MI models (32). The addition of anti-CD8 antibodies significantly reduced the number of cardiomyocytes destroyed by lymphocytes post-MI (33). The present data revealed that VCP979 treatment also markedly decreased the infiltration of $\mathrm{CD}^{+} \mathrm{T}$-cells in MI/R hearts on day 14 .

Macrophages are equally pivotal for local infarct zone remodeling, as they are involved in the initiation and resolution phases of cardiac inflammatory cascades (34). Preclinical data has shown that increased cardiac wall tension may stimulate local macrophage proliferation following heart failure (35). There are two typical subtypes of macrophages post-MI/R injury: Classically activated M1 macrophages and alternatively activated M2 macrophages (36). M1 to M2 macrophage transition improved heart repair and M2 macrophages promoted scar formation and neo-vascularization (37). Arg1, a M2 polarization marker, promoted an anti-inflammatory reaction by downregulating M1 markers (iNOS) following MI (38). In the present study, VCP979 treatment markedly decreased the infiltration of $\mathrm{F} 4 / 80^{+}$macrophages in MI/R hearts. In addition, a downregulation of M1 markers (iNOS) and an elevated expression of M2 polarization markers (Arg1, CD206) were identified in reperfused myocardia on day 14 . Collectively, the present results indicated that VCP979 may protect the heart from MI/R injury by inhibiting T cell- and macrophage-medicated inflammatory response.

Upon infarction, specific inflammatory responses are activated by cellular effectors, including immune cells, cardiomyocytes, fibroblasts and vascular cells which secrete plenty of pro/anti-inflammatory cytokines and chemokines (39). Pro-inflammatory cytokines and chemokines are associated with the pathogenesis of heart repair and ventricular remodeling in MI/R injury progression (25). Pro-inflammatory cytokines, including IL-1 $\beta$, IL-6, IL-8, TNF- $\alpha$ and mitochondrial pyruvate carrier-1, are secreted and upregulated from adjacent cardiomyocytes, resulting in leukocyte infiltration and infarct area expansion (10). Other cytokines, such as interferon- $\gamma$, IL- 2 and IL-4, are secreted by T cells and further stimulate the chemotaxis of monocytes and neutrophils in MI/R injury (40). Conversely, inhibitory cytokines, such as IL-10, are involved in the suppression of extracellular matrix formation and pro-inflammatory cytokine secretion (38). IL-4 and IL-13 may also repress inflammatory responses and induce macrophage deactivation post-MI/R injury (38). It was found that VCP979 reduced the mRNA levels of pro-inflammatory cytokines in reperfusion myocardia on day 14 following MI injury. Therefore, these results indicated that VCP979 may contribute to cardiac protection by inhibiting the inflammatory response.

Ang II is a key biologically active component of the renin-Ang system and is produced by the hydrolysis of Ang I under the action of Ang-converting enzyme (24). Previous studies have reported that Ang II can modulate the proliferation and activity of cardiac fibroblasts, and stimulate collagen synthesis $(41,42)$. In addition, Ang II may directly promote cell hypertrophy and accelerate protein synthesis and cell size in vitro (41). Clinical studies verified that the plasma Ang II level was elevated in heart failure patients, which may be associated with cardiac dysfunction and poor prognosis $(43,44)$. Ang II can directly regulate downstream inflammatory activities through several signaling pathways, such as phospho-p38 (45). It was found that, in murine MI/R injury models, the mRNA level of Ang II markedly increased in hearts on day 28, but VCP979 treatment reversed this elevation. Consistent with previous reports $(41,46)$, the addition of Ang II induced significant collagen synthesis in NCFs and hypertrophy in NCMs. The present results further revealed that the addition of VCP979 prevented NCFs from Ang II-induced collagen synthesis and NCMs from Ang II-induced hypertrophy in the cell culture system.

MAPKs signaling pathways, particularly p38-MAPK, play crucial roles in the MI/R injury and consequent pathological remodeling (9). Preclinical evidence has shown that myocardial ischemia is a potent stimulant of p38 activation, which might increase the production of inflammatory cytokines and accelerate the rate of infarction/death (9). A previous study has demonstrated that the p38-MAPK signaling pathway inhibitor SB203580 significantly repressed MI/R injury through its anti-inflammatory, anti-oxidative and anti-apoptosis effects (47). In the present experiments, the small-molecule compound VCP979 inhibited the MI/R injury-triggered activation of the p38-MAPK signaling pathway in murine models. In addition, VCP979 markedly reduced NCFs collagen synthesis exposed to Ang II by suppressing the p38-MAPK signaling pathway in vitro. Although the present study demonstrated that VCP979 inhibited myocyte hypertrophy, whether VCP979 exerted its roles on phosphorylated p38 MAPK in NCMs needs further investigation. 
The present study, to the best of our knowledge was the first to demonstrate that the novel small-molecule compound VCP979 may improve ventricular remodeling in murine MI/R injury models by ameliorating pathological myocardial fibrosis and hypertrophy. The protective effects of VCP979 in murine MI/R injury models were attributed to the inhibition of the inflammatory response and the suppression of the p38-MAPK signaling pathway. Therefore, VCP979 may serve as a small-molecule drug for the clinical treatment of $\mathrm{MI} / \mathrm{R}$ injury. Future clinical studies are required to verify its efficacy and prospects.

\section{Acknowledgements}

The authors would like to thank Dr Liang Zheng from the Research Center for Translational Medicine, Shanghai East Hospital, Tongji University School of Medicine, for statistical assistance.

\section{Funding}

The present study was supported by the National Nature Science Foundation of China (NSFC; grant nos. 81670458,81470393 and 81370434), Shanghai Municipal Health and Family Planning Commission [grant nos. ZY(2018-2020)-FWTX-2007], Shanghai Key Medical Discipline for Critical Care Medicine (grant nos. 2017zz02017), The National Key Research and Development Program of China (grant nos. 2017YFA0105600), The Science and Technology Commission of Shanghai Municipality (grant nos. 17431906600), Three-year plan on TCM of Pudong Health Bureau of Shanghai (grant nos. PDZY-2018-0603) and the National Health and Medical Research Council of Australia (program grant no. 1092642 and development grant no. 546243 to BHW).

\section{Availability of data and materials}

All data generated or analyzed during this study are included in this paper, and more detailed data in the current study are available from the corresponding author on reasonable request.

\section{Authors' contributions}

$\mathrm{ZL}, \mathrm{XZ}$ and $\mathrm{BW}$ contributed to the conception and design of the study. JL, QM, XL, RZ, DY, XG, HC, FL, XG, HF contributed to acquisition, analysis and interpretation of the data. JL, $\mathrm{XZ}, \mathrm{ZL}$ and BW wrote and revised the manuscript. All authors read and approved the final manuscript.

\section{Ethics approval and consent to participate}

The experimental procedures were implemented following the approval of the Institutional Animal Care and Use Committee of Tongji University (Shanghai, China; approval no. TJLAC-019-121).

\section{Patient consent for publication}

Not applicable.

\section{Competing interests}

The authors declare that they have no competing interests.

\section{References}

1. Moran AE, Forouzanfar MH, Roth GA, Mensah GA, Ezzati M, Flaxman A, Murray CJ and Naghavi M: The global burden of ischemic heart disease in 1990 and 2010: The global burden of disease 2010 study. Circulation 129: 1493-1501, 2014.

2. Miura T and Miki T: Limitation of myocardial infarct size in the clinical setting: Current status and challenges in translating animal experiments into clinical therapy. Basic Res Cardiol 103: 501-513, 2008.

3. Eltzschig HK and Eckle T: Ischemia and reperfusion-from mechanism to translation. Nat Med 17: 1391-1401, 2011.

4. Li Y, Li Z, Zhang C, Li P, Wu Y, Wang C, Bond Lau W, Ma XL and Du J: Cardiac fibroblast-specific activating transcription factor 3 protects against heart failure by suppressing MAP2K3-p38 signaling. Circulation 135: 2041-2057, 2017.

5. Krum H and Teerlink JR: Medical therapy for chronic heart failure. Lancet 378: 713-721, 2011.

6. Kloner RA: Current state of clinical translation of cardioprotective agents for acute myocardial infarction. Circ Res 113: 451-463, 2013.

7. Gerczuk PZ and Kloner RA: An update on cardioprotection: A review of the latest adjunctive therapies to limit myocardial infarction size in clinical trials. J Am Coll Cardiol 59: 969-978, 2012.

8. Arkin MR and Wells JA: Small-molecule inhibitors of protein-protein interactions: Progressing towards the dream. Nat Rev Drug Discov 3: 301-317, 2004.

9. Kumphune S, Chattipakorn S and Chattipakorn N: Role of p38 inhibition in cardiac ischemia/reperfusion injury. Eur J Clin Pharmacol 68: 513-524, 2012

10. Prompunt E, Sanit J, Barrère-Lemaire S, Nargeot J, Noordali H, Madhani M and Kumphune S: The cardioprotective effects of secretory leukocyte protease inhibitor against myocardial ischemia/reperfusion injury. Exp Ther Med 15: 5231-5242, 2018.

11. Cai Y, Lu C, Xu T, Ma Y, Min S, Scammells P, Wang B and Ju S: Diffusion tensor imaging evaluation of axonal/white matter remodeling in a mouse model of diabetic stroke treated with novel p38 MAPK inhibitor, VCP979. J Biomed Nanotechnol 14: 585-593, 2018

12. Vinh NB, Devine SM, Munoz L, Ryan RM, Wang BH, Krum H, Chalmers DK, Simpson JS and Scammells PJ: Design, synthesis, and biological evaluation of tetra-substituted thiophenes as inhibitors of p38 $\alpha$ MAPK. ChemistryOpen 4: 56-64, 2015.

13. Xu T, Ding J, Ge H, Xu X, Scammells P, Wang B and Ju S: Effects of VCP979 novel p38 mitogen activated protein kinase inhibitor on progression of pancreatic cancer in mouse model with diabetic conditions. J Biomed Nanotechnol 15: 1325-1333, 2019.

14. Yuan D, Tie J, Xu Z, Liu G, Ge X, Wang Z, Zhang X, Gong S, Liu G, Meng Q, et al: Dynamic profile of CD4(+) T-cell-associated cytokines/chemokines following murine myocardial infarction/reperfusion. Mediators Inflamm 2019: 9483647, 2019.

15. Yang L, Wang B, Zhou Q, Wang Y, Liu X, Liu Z and Zhan Z: MicroRNA-21 prevents excessive inflammation and cardiac dysfunction after myocardial infarction through targeting KBTBD7. Cell Death Dis 9: 769, 2018.

16. Zhu J, Ye Q, Xu S, Chang YX, Liu X, Ma Y, Zhu Y and Hua S: Shengmai injection alleviates $\mathrm{H} 2 \mathrm{O} 2$ induced oxidative stress through activation of AKT and inhibition of ERK pathways in neonatal rat cardiomyocytes. J Ethnopharmacol 239: 111677, 2019.

17. Dolber PC, Beyer EC, Junker JL and Spach MS: Distribution of gap junctions in dog and rat ventricle studied with a double-label technique. J Mol Cell Cardiol 24: 1443-1457, 1992.

18. Zhuang R, Wu J, Lin F, Han L, Liang X, Meng Q, Jiang Y, Wang Z, Yue A, Gu Y, et al: Fasudil preserves lung endothelial function and reduces pulmonary vascular remodeling in a rat model of endstage pulmonary hypertension with left heart disease. Int $\mathrm{J}$ Mol Med 42: 1341-1352, 2018.

19. Dick SA and Epelman S: Chronic heart failure and inflammation: What do we really know? Circ Res 119: 159-176, 2016.

20. Tamargo J, Caballero R and Delpón E: New drugs in preclinical and early stage clinical development in the treatment of heart failure. Expert Opin Investig Drugs 28: 51-71, 2019. 
21. Lemke LE, Bloem LJ, Fouts R, Esterman M, Sandusky G and Vlahos CJ: Decreased p38 MAPK activity in end-stage failing human myocardium: p38 MAPK alpha is the predominant isoform expressed in human heart. J Mol Cell Cardiol 33: 1527-1540, 2001.

22. Shinde AV and Frangogiannis NG: Fibroblasts in myocardial infarction: A role in inflammation and repair. $\mathrm{J}$ Mol Cell Cardiol 70: 74-82, 2014.

23. Kaur H, Takefuji M, Ngai CY, Carvalho J, Bayer J, Wietelmann A, Poetsch A, Hoelper S, Conway SJ, Möllmann H, et al: Targeted ablation of periostin-expressing activated fibroblasts prevents adverse cardiac remodeling in mice. Circ Res 118: 1906-1917, 2016.

24. Ma Y, Hu Y, Wu J, Wen J, Li S, Zhang L, Zhang J, Li Y and Li J: Epigallocatechin-3-gallate inhibits angiotensin II-induced cardiomyocyte hypertrophy via regulating Hippo signaling pathway in H9c2 rat cardiomyocytes. Acta Biochim Biophys Sin (Shanghai) 51: 422-430, 2019.

25. French BA and Kramer CM: Mechanisms of post-infarct left ventricular remodeling. Drug Discov Today Dis Mech 4: 185-196, 2007.

26. Kompa AR, See F, Lewis DA, Adrahtas A, Cantwell DM, Wang $\mathrm{BH}$ and Krum H: Long-term but not short-term p38 mitogen-activated protein kinase inhibition improves cardiac function and reduces cardiac remodeling post-myocardial infarction. J Pharmacol Exp Ther 325: 741-750, 2008.

27. Di Napoli P, Taccardi AA, De Caterina R and Barsotti A: Pathophysiology of ischemia-reperfusion injury: Experimental data. Ital Heart J 3 (Suppl 4): 24s-28s, 2002.

28. Hofmann U and Frantz S: Role of lymphocytes in myocardial injury, healing, and remodeling after myocardial infarction. Circ Res 116: 354-367, 2015.

29. Hofmann U, Beyersdorf N, Weirather J, Podolskaya A, Bauersachs J, Ertl G, Kerkau T and Frantz S: Activation of CD4+ $\mathrm{T}$ lymphocytes improves wound healing and survival after experimental myocardial infarction in mice. Circulation 125 : 1652-1663, 2012.

30. Boag SE, Andreano E and Spyridopoulos I: Lymphocyte communication in myocardial ischemia/reperfusion injury. Antioxid Redox Signal 26: 660-675, 2017.

31. Hofmann U and Frantz S: Role of T-cells in myocardial infarction. Eur Heart J 37: 873-879, 2016.

32. Stabile E, Kinnaird T, la Sala A, Hanson SK, Watkins C, Campia U, Shou M, Zbinden S, Fuchs S, Kornfeld H, et al: CD8+ T lymphocytes regulate the arteriogenic response to ischemia by infiltrating the site of collateral vessel development and recruiting CD4+ mononuclear cells through the expression of interleukin-16. Circulation 113: 118-124, 2006.

33. Varda-Bloom N, Leor J, Ohad DG, Hasin Y, Amar M, Fixler R, Battler A, Eldar M and Hasin D: Cytotoxic T lymphocytes are activated following myocardial infarction and can recognize and kill healthy myocytes in vitro. J Mol Cell Cardiol 32: 2141-2149, 2000.

34. Panizzi P, Swirski FK, Figueiredo JL, Waterman P, Sosnovik DE, Aikawa E, Libby P, Pittet M, Weissleder R and Nahrendorf M: Impaired infarct healing in atherosclerotic mice with Ly-6C(hi) monocytosis. J Am Coll Cardiol 55: 1629-1638, 2010.
35. Sager HB, Hulsmans M, Lavine KJ, Moreira MB, Heidt T, Courties G, Sun Y, Iwamoto Y, Tricot B, Khan OF, et al: Proliferation and recruitment contribute to myocardial macrophage expansion in chronic heart failure. Circ Res 119: 853-864, 2016.

36. Zhao J, Li X, Hu J, Chen F, Qiao S, Sun X, Gao L, Xie J and Xu B: Mesenchymal stromal cell-derived exosomes attenuate myocardial ischemia-reperfusion injury through miR-182-regulated macrophage polarization. Cardiovasc Res 115: 1205-1216, 2019.

37. Prabhu SD: It takes two to tango: Monocyte and macrophage duality in the infarcted heart. Circ Res 114: 1558-1560, 2014.

38. Frangogiannis NG, Mendoza LH, Lindsey ML, Ballantyne CM, Michael LH, Smith CW and Entman ML: IL-10 is induced in the reperfused myocardium and may modulate the reaction to injury. J Immunol 165: 2798-2808, 2000.

39. Prabhu SD and Frangogiannis NG: The biological basis for cardiac repair after myocardial infarction: From inflammation to fibrosis. Circ Res 119: 91-112, 2016.

40. Yang Z, Day YJ, Toufektsian MC, Xu Y, Ramos SI, Marshall MA, French BA and Linden J: Myocardial infarct-sparing effect of adenosine A2A receptor activation is due to its action on CD4+ T lymphocytes. Circulation 114: 2056-2064, 2006.

41. Iwata M, Cowling RT, Yeo SJ and Greenberg B: Targeting the ACE2-Ang-(1-7) pathway in cardiac fibroblasts to treat cardiac remodeling and heart failure. J Mol Cell Cardiol 51: 542-547, 2011.

42. Song Q, Liu L, Yu J, Zhang J, Xu M, Sun L, Luo H, Feng Z and Meng G: Dihydromyricetin attenuated Ang II induced cardiac fibroblasts proliferation related to inhibitory of oxidative stress. Eur J Pharmacol 807: 159-167, 2017.

43. Iwata M, Cowling RT, Gurantz D, Moore C, Zhang S, Yuan JX and Greenberg BH: Angiotensin-(1-7) binds to specific receptors on cardiac fibroblasts to initiate antifibrotic and antitrophic effects. Am J Physiol Heart Circ Physiol 289: H2356-H2363, 2005.

44. Basu R, Poglitsch M, Yogasundaram H, Thomas J, Rowe BH and Oudit GY: Roles of angiotensin peptides and recombinant human ACE2 in heart failure. J Am Coll Cardiol 69: 805-819, 2017.

45. Shatanawi A, Romero MJ, Iddings JA, Chandra S, Umapathy NS, Verin AD, Caldwell RB and Caldwell RW: Angiotensin II-induced vascular endothelial dysfunction through RhoA/Rho kinase/p38 mitogen-activated protein kinase/arginase pathway. Am J Physiol Cell Physiol 300: C1181-C1192, 2011.

46. Du M, Huang K, Gao L, Yang L, Wang WS, Wang B, Huang K and Huang D: Nardosinone protects $\mathrm{H} 9 \mathrm{c} 2$ cardiac cells from angiotensin II-induced hypertrophy. J Huazhong Univ Sci Technolog Med Sci 33: 822-826, 2013.

47. Zhang X, Wang Y, Shen W, Ma S, Chen W and Qi R: Rosa rugosa flavonoids alleviate myocardial ischemia reperfusion injury in mice by suppressing JNK and p38 MAPK. Microcirculation 24: 2017.

This work is licensed under a Creative Commons Attribution-NonCommercial-NoDerivatives 4.0 International (CC BY-NC-ND 4.0) License. 